\title{
ERRATUM
}

\section{Limits on classical communication from quantum entropy power inequalities}

Robert König \& Graeme Smith

Nature Photon. 7, 142-146 (2013); published online 20 January 2013; corrected after print 24 January 2013.

In the version of this Article originally published, the author affiliations were incorrect and should have read: ${ }^{1} \mathrm{IBM}$ TJ Watson Research Center, 1101 Kitchawan Road, Yorktown Heights, New York 10598, USA, ${ }^{2}$ Institute for Quantum Computing and Department of Applied Mathematics, University of Waterloo, ON, Canada, N2L3G1.

This has now been corrected in the HTML and PDF versions of the Article. 